\title{
Displasia ectodérmica hipohidrótica. Revisión de sus manifestaciones otorrinolaringológicas a propósito de dos casos
}

\author{
Hypohidrotic ectodermal dysplasia. \\ Review of ENT manifestations on purpose of two cases
}

René Sepúlveda S', Daniel Muñoz S², Felipe Cardemil M', Maritza Rahal E.

\begin{abstract}
RESUMEN
La displasia ectodérmica hipohidrótica es una enfermedad hereditaria, infrecuente en la población general, caracterizada clínicamente por la tríada de hipohidrosis, deficiencias dentarias y falta de crecimiento de cabello. Además, se afectan otros anexos cutáneos como folículos pilosos, uñas y dientes e incluso estructuras como las glándulas mucosas del tracto aerodigestivo superior lo que puede ocasionar signos, síntomas y patologías de interés para el otorrinolaringólogo.

Se presentan dos casos clínicos, un adulto y un niño, y se revisa la literatura respecto de las manifestaciones de la displasia ectodérmica hipohidrótica en cabeza y cuello.
\end{abstract}

Palabras clave: Displasia ectodérmica hipohidrótica, ocena, epistaxis.

\begin{abstract}
Hypohidrotic ectodermal dysplasia is an uncommon, hereditary disease, clinically characterized by the triad of hypohidrosis, dental deficiencies and lack of hair growth. In addition, it affects other skin appendages such as hair follicles, nails and teeth and even structures such as the mucous glands of the upper aerodigestive tract causing signs and also symptoms and pathologies of interest for the otolaryngologist.

Two cases are presented, an adult and a child one, an in addition to literature review regarding the manifestations of hypohidrotic ectodermal dysplasia in head and neck.

Key words: Hypohidrotic ectodermal dysplasia, ozena, epistaxis.

\section{INTRODUCCIÓN}

La displasia ectodérmica hipohidrótica (DEH) o síndrome de Christ-Siemens-Touraine es la forma más frecuente de las displasias ectodérmicas y su prevalencia se estima en torno a 1/100.000 recién nacidos ${ }^{2}$. Es una enfermedad hereditaria ligada al sexo (cromosoma X), caracterizada clínicamente por la triada de
\end{abstract}

La displasia ectodérmica agrupa una gran variedad de cuadros clínicos que comparten rasgos comunes como el compromiso de dos o más de las siguientes estructuras: Pelo, dientes, uñas, glándulas sudoríparas y otras estructuras ectodérmicas ${ }^{1}$.

Médico. Servicio de Otorrinolaringología, Hospital Barros Luco-Trudeau.

2 Médico. Universidad de Chile. 
hipohidrosis, deficiencias dentarias y falta de crecimiento de cabello ${ }^{3}$.

La disminución del número y tamaño de las glándulas sudoríparas produce sequedad cutánea y en ocasiones crisis de hiperpirexia ${ }^{4}$. Además, como se mencionó, también se afectan otros anexos cutáneos como folículos pilosos, uñas y dientes e incluso estructuras como las glándulas mucosas del tracto aerodigestivo superior lo que puede ocasionar signos, síntomas y patologías de interés para el otorrinolaringólogo5.

\section{PRESENTACION DE CASOS}

\section{Caso 1}

Paciente de 52 años de edad, con antecedentes de hipertensión arterial, diabetes mellitus tipo 2, cirugía nasal por puente bajo a los 7 años y una hospitalización el año 1988 por quiste hidatídico pulmonar derecho operado. En aquella oportunidad tuvo epistaxis que se manejó con taponamiento posterior.

Paciente es hospitalizado por cuadro de epistaxis por fosa nasal izquierda, que no logra ser manejada con taponamiento nasal anterior, instalándose un taponamiento posterior. El examen endonasal demuestra atrofia de cornetes, sangrado septal posterior y perforación del mismo, con abundantes costras.

Destaca también al examen físico frente amplia, hipotricosis, hipertelorismo, puente nasal bajo, nariz pequeña, vestíbulos nasales estrechos, edentado parcial superior e inferior de incisivos, piel seca y presión arterial elevada (Figuras 1 y 2).
Dirigidamente, refiere presentar cacosmia asociado a costras abundantes mayores a izquierda desde la adolescencia. Además describe en su historia que no presenta sudoración y sufrir sensación de calor y en ocasiones fiebre, sin causa aparente. Tuvo 2 episodios de epistaxis en los últimos 4 años, que se han manejado con taponamiento anterior en la atención primaria.

Se realiza tomografía computarizada de cavidades paranasales, donde destaca hipoplasia de seno maxilar izquierdo y agenesia dentaria (Figuras 3 y 4 ).

Se retira taponamiento en pabellón al octavo día. Se visualizan pólipos etmoidales que se resecan, atrofia de cornetes, desviación septal derecha

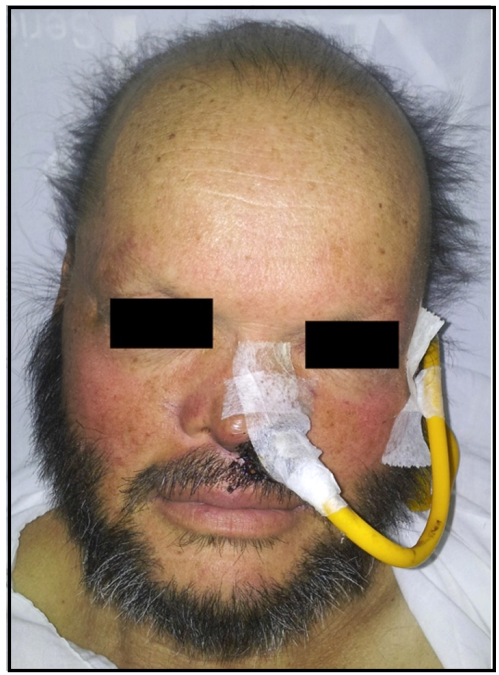

Figura 1. Fascies del paciente del caso clínico 1, con taponamiento posterior instalado, destaca su frente amplia, hipotricosis e hipertelorismo.

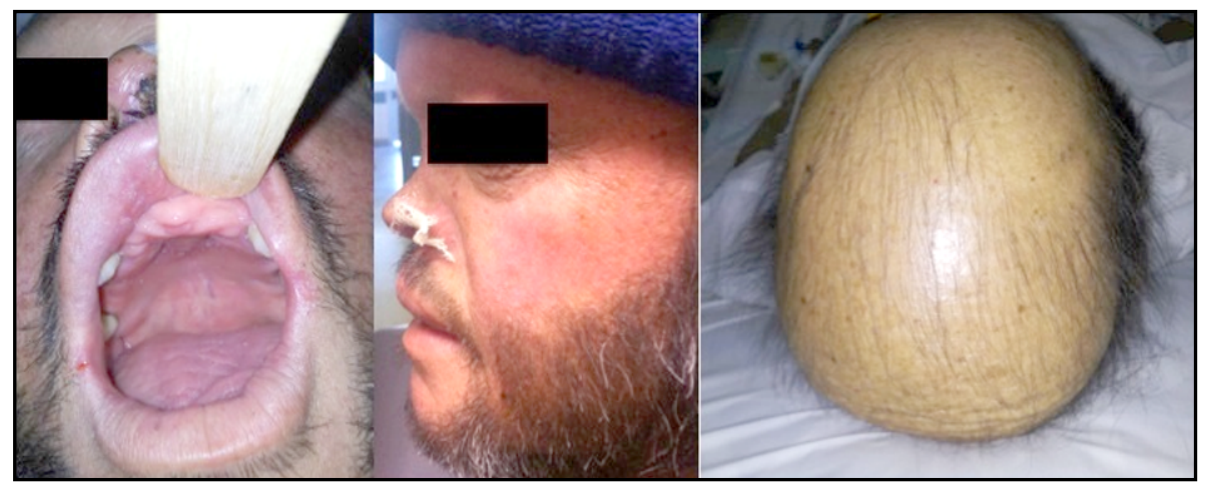

Figura 2. Fotografías del caso clínico 1, destacando algunas de sus características clínicas más relevantes: hipodoncia, puente nasal bajo e hipotricosis. 


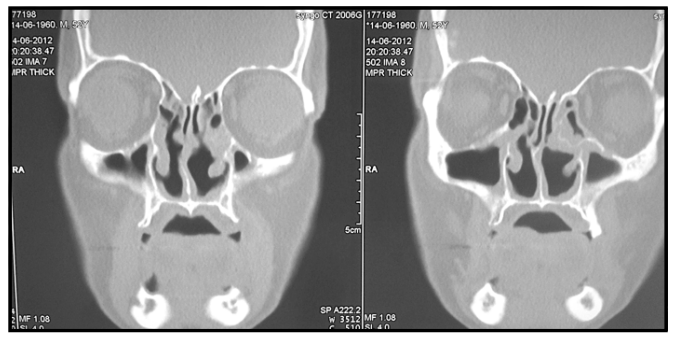

Figura 3. Tomografía computarizada de cavidades paranasales, corte coronal, destaca la hipoplasia de los senos paranasales, mayor a izquierda, en paciente con displasia ectodérmica.

con perforación hacia posterior y sangrado escaso. No se constató en los exámenes de laboratorio ninguna alteración en la coagulación. Se realiza biopsia de piel, que muestra disminución de la cantidad de glándulas sudoríparas y ausencia parcial de los folículos pilosos y glándulas sebáceas.

\section{Caso 2}

Paciente de sexo masculino, 2 años de edad, diagnosticado de displasia ectodérmica hipohidrótica. Consulta por historia de epistaxis recurrente, secreción nasal y halitosis. Al examen físico destaca frente amplia, hipotricosis, hipertelorismo, puente nasal bajo, nariz pequeña, vestíbulos nasales estrechos y piel seca. Se realiza nasofibroscopía que evidencia ocena, presencia de costras en cavidad nasal y a nivel laríngeo eritema y edema de aritenoides. Se toma cultivo de costras en cavidad nasal que demuestra presencia de Stafilococcus aureus, que se erradica según indicación de infectología. Se maneja medicamente el reflujo faringolaríngeo y se indica aseo nasal con solución salina, con buena respuesta y sin nuevos episodios de epistaxis.

\section{DISCUSIÓN}

La displasia ectodérmica es una enfermedad genética que se caracteriza por la afectación de estructuras que derivan del ectodermo. La patogenia se explica por la alteración que se produce durante el tercery cuarto mes de gestación en la maduración de algunos tejidos ectodérmicos ${ }^{6}$.

El cuadro clínico se caracteriza por alteración de la piel, anexos cutáneos y otros derivados del ectodermo. La piel es seca, con disminución de

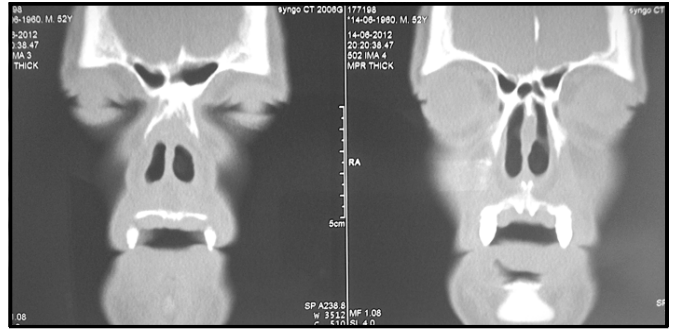

Figura 4. Tomografia computarizada de senos paranasales, corte coronal que evidencia ausencia de desarrollo de los incisivos superiores, en paciente con displasia ectodérmica.

folículos pilosos y escasa presencia de glándulas sudoríparas, lo que produce hipohidrosis y alteración de la regulación de la temperatura corporal. También pueden presentarse anomalías ungueales, hipotricosis, hipodoncia. El compromiso de las glándulas mucosas del tracto aerodigestivo superior ${ }^{2,7}$,da origen a alteraciones otorrinolaringológicas, que a continuación revisamos (Tabla 1).

\section{Oído}

Se ha descrito una anomalía de pabellón auricular característica, con hipoplasia de la concha y del lóbulo auricular. La migración de la descamación del epitelio

\section{Tabla 1. Resumen de manifestaciones otorrinolaringológicas \\ de la displasia ectodérmica hipohidrótica}

\begin{tabular}{|ll|}
\hline Otológicas & Tapón de cerumen \\
& Otitis externa \\
& Eczema de conducto auditivo \\
& Otitis media crónica serosa \\
Nasosinusales & Hipoacusia sensorioneural \\
& Rinitis crónica (ocena) \\
& Epistaxis \\
& Deformidad en silla de montar \\
& Rinosinusitis aguda/recurrente/crónica \\
& Hipoplasia seno maxilar \\
Cavidad oral & Hipodoncia \\
Faringe y laringe & $\begin{array}{l}\text { Xerostomía } \\
\text { Disfagia faríngea } \\
\text { Laringitis crónica } \\
\text { Tráquea y bronquios }\end{array}$ \\
& $\begin{array}{l}\text { Nódulos vocales } \\
\text { Infecciones respiratorias recurrentes } \\
\text { Carcinoma escamoso traqueal }\end{array}$ \\
\end{tabular}


en el conducto auditivo externo está alterado en estos pacientes, lo que da lugar a tapones de cerumen, otitis externas y eczema del conducto auditivo externo ${ }^{8}$.

Por otro lado, la glándulas mucosas hipoplásicas 0 ausentes alteran la función de la trompa de Eustaquio, por lo que los pacientes pueden desarrollar otitis media crónica serosa. Se han reportado también casos de hipoacusia sensorioneural en estos pacientes, no existiendo claridad sobre su mecanismo9.

\section{Nariz y cavidades paranasales}

Las manifestaciones nasales descritas son rinitis crónica (ocena), siendo esta la más frecuente según varias series ${ }^{5-7}$. Además, deformidad en silla de montar, formación de costras debido a secreciones espesas y un transporte mucociliar ineficaz que lleva a la ectasia de moco, con infección secundaria de senos paranasales y fosas nasales, y epistaxis ${ }^{10}$. Estos pacientes, como los nuestros, están predispuestos a padecer epistaxis con cierta frecuencia debido a la sequedad de la mucosa y la presencia de costras. En este sentido, es de extrema utilidad el uso regular de ungüentos y lavados nasales ${ }^{11}$. En nuestro caso además, es posible apreciar hipoplasia del seno maxilar.

\section{Cavidad oral}

Las manifestaciones clásicas son hipodoncia y sequedad bucal. Esta combinación, puede afectar la nutrición, articulación de la palabra y obviamente su apariencia, por lo que es perentoria la evaluación odontológica en estos pacientes. También se asocia a fisura labio-palatina ${ }^{12}$.

\section{Faringe y laringe}

La falta se secreción salival puede provocar disfagia a nivel faríngeo. La manifestación laríngea clásica es la disfonía por laringitis crónica atribuible a la falta de lubricación de las cuerdas vocales. La humidificación de la vía aérea y terapia vocales de utilidad, sobre todo en aquellos casos que se asocie a nódulos vocales, como se ha descrito ${ }^{13}$.

\section{Tráquea y bronquios}

Debido al espesamiento del mucus y alteración de la función ciliar, se asocia la DEH con infecciones de la vía aérea, lo que sumado a la presencia de hipogamaglobulinemia en estos pacientes, explicaría el aumento de la frecuencia de infecciones del tracto aerodigestivo superior, bronquitis y neumonías ${ }^{14}$. Existe incluso, un reporte de asociación entre carcinoma de células escamosas en tráquea y displasia ectodérmica ${ }^{15}$.

\section{CONCLUSIONES}

La displasia ectodérmica es una enfermedad infrecuente, con manifestaciones diversas como hipohidrosis, rinitis crónica, hipodoncia, uñas distróficas, alopecia y facie característica. La mayor parte de las manifestaciones de la displasia ectodérmica, como resultado de la ausencia 0 hipoplasia de las glándulas mucosas del tracto aerodigestivo superior, se presentan en cabeza y cuello, por lo que es de interés en nuestra especialidad conocerla. El papel del otorrinolaringólogo incluye lubricación nasal, manejo de la epistaxis, tratamiento de complicaciones infecciosas y tratamiento de la hipoacusia.

\section{BIBLIOGRAFÍA}

1. Cummings Otolaryngology: Head \& Neck Surgery. $5^{\text {th }}$ ed. Philadelphia, Pa: Mosby Elsevier; 2010.

2. Gorlin R, Cohen M, Hennekam R. Syndromes of the Head and Neck. Oxford Monographs on Medical Genetics, 2010. Oxford University Press, USA; $5^{\text {th }}$ edition.

3. Ibañes Cárcamo E. Anhidrotic ectodermal dysplasia: congenital ozena. Acta Otorrinolaringol Esp 1990; 41(2): 107-9.

4. Berg D, Weingold DH, Abson KG, Olsen EA. Sweating in ecto- dermal dysplasias syndromes. A review. Arch Dermatol 1990; 126: 1075-9.

5. González García J, Galera Ruiz H, Muñoz Borge F. ENT expression of hypohidrotic ectodermal dysplasia. Acta Otorrinolaringol Esp 2005; 56(4): 176-8.

6. Ezzine Sebai N, Khaled A, Kharfi M, Bouzgarrou A, M'halla H, Jones M, Fazaa B, Kamoun MR. Anhidrotic/hypohidrotic ectodermal dysplasia: ten cases. Tunis Med 2009; 87(12): 805-9. 
7. Daniel E, McCurdy EA, Shashi V, McGuirt WF Jr. Ectodermal dysplasia: otolaryngologic manifestations and management. Laryngoscope 2002; 112(6): 962-7.

8. ShIN JJ, HARTNICK CJ. Otologic manifestations of ectodermal dysplasia. Arch Otolaryngol Head Neck Surg 2004; 130(9): 1104-7.

9. Yildirim M, Yorgancilar E, Gun R, Topcu I. Ectodermal dysplasia:otolaryngologic evaluation of 23 cases. Ear Nose Throat J 2012; 91(2): E2833.

10. Barman D, Mandal S, Nandi S, Baneruee P, Rashid MA. Anhidrotic ectodermal dysplasia presenting as atrophic rhinitis. J Indian Med Assoc 2011; 109(11): 836-7.

11. Mehta U, Brunworth J, Lewis Ra, Sindwani R. Rhinologic manifestations of ectodermal dysplasia. Am J Rhinol 2007; 21(1): 55-8.
12. de Aquino SN, Paranaíba LM, Swerts MS, Martelli DR, dE BARRos LM, JúNIOR HM. Orofacial Features of Hypohidrotic Ectodermal Dysplasia. Head Neck Pathol 2012 Mar 16. Epub ahead of print.

13. Mehta U, Brunworth J, Fete TJ, Sindwani R. Head and neck manifestations and quality of life of patients with ectodermal dysplasia. Otolaryngol Head Neck Surg 2007; 136(5): 843-7.

14. Winter SC, Bates GJ. Hypohidrotic ectodermal dysplasia associated with squamous cell carcinoma of the trachea. J Laryngol Otol 2002; 116: 742-3.

15. Mark BJ, Becker Ba, Halloran DR, Bree AF, Sindwani R, Fete MD, Motil KJ, Srun SW, Fete TJ. Prevalence of atopic disorders and immunodeficiency in patients with ectodermal dysplasia syndromes. Ann Allergy Asthma Immunol 2012; 108(6): 435-8. 